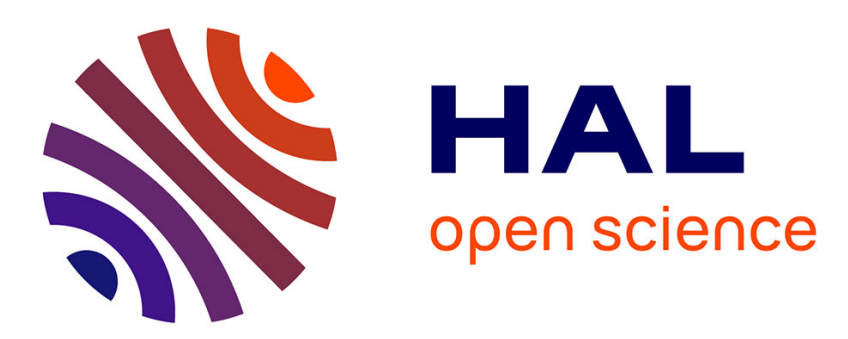

\title{
Nouveaux abaques pour la détermination des constantes diélectriques complexes aux hyperfréquences
}

\author{
G. Delbos, C. Demau
}

\section{To cite this version:}

G. Delbos, C. Demau. Nouveaux abaques pour la détermination des constantes diélectriques complexes aux hyperfréquences. Revue de Physique Appliquée, 1966, 1 (4), pp.287-292. 10.1051/rphysap:0196600104028700 . jpa-00242735

\section{HAL Id: jpa-00242735 https://hal.science/jpa-00242735}

Submitted on 1 Jan 1966

HAL is a multi-disciplinary open access archive for the deposit and dissemination of scientific research documents, whether they are published or not. The documents may come from teaching and research institutions in France or abroad, or from public or private research centers.
L'archive ouverte pluridisciplinaire HAL, est destinée au dépôt et à la diffusion de documents scientifiques de niveau recherche, publiés ou non, émanant des établissements d'enseignement et de recherche français ou étrangers, des laboratoires publics ou privés. 


\title{
NOUVEAUX ABAQUES POUR LA DÉTERMINATION DES CONSTANTES DIÉLECTRIQUES COMPLEXES AUX HYPERFRÉQUENCES
}

\author{
Par G. DELBOS et C. DEMAU, \\ Laboratoire d'Optique Ultrahertzienne de la Faculté des Sriences de Bordeaux. \\ Equipe de recherche associée au C. N. R. S.
}

\begin{abstract}
Résumé. - On expose, pour la détermination des constantes diélectriques complexes aux hyperfréquences, un nouveau mode d'exploitation de la méthode dite " de la ligne courtcircuitée ". Il consiste en la réalisation d'abaques nouveaux à lectures directes donnant $\varepsilon^{\prime}$ et $\varepsilon^{\prime \prime}$ à partir du TOST et du déplacement d'un minimum du train d'ondes stationnaires et en une méthode d'affinement et de séparation des mesures, pour la détermination des faibles absorptions.
\end{abstract}

Abstract. - A new way of operating the slotted-line method for the determination of complex dielectric constants is described. It consist in the construction of new charts giving $\varepsilon^{\prime}$ and $\varepsilon^{\prime \prime}$ from VSWR and position of the voltage minimum, and a method of refining and separation of the mesurements for the cietermination of low losses.

\section{Description et utilisation des abaques. -} 1.1. Introduction. - Les techniques de mesures des constantes diélectriques complexes aux hyperfréquences sont très variées [1], [2], [3], [4] et correspondent chacune à des domaines de mesures différents. Pour des mesures absolues la méthode dite de la "ligne court-circuitée » reste la plus utilisée. Malheureusement jusqu'à présent son utilisation était rendue très délicate du fait des nombreux calculs, pourtant déjà bien simplifiés par l'utilisation de diagrammes (Smith) et d'abaques [5], [6], [7].

Il était donc intéressant de réenvisager cette méthode avec des moyens de calculs rapides (Ordinateur I. B. M. 1620) en considérant la permittivité complexe $\varepsilon$ en fonction directe des données expérimentales.

Cela nous a conduit d'abord à tracer un système de quatre abaques permettant par simple lecture d'apprécier avec une bonne précision les valeurs des parties réelle et imaginaire de la constante diélectrique complexe [10].

Nous allons donner une description complète de ces abaques et leur utilisation après avoir fait un bref rappel théorique de la méthode de la ligne court-circuitée.

En ce qui concerne l'utilisation de ces abaques, il nous a paru indispensable de rappeler la technique des mesures de TOST le long d'une ligne à pertes.

Nous donnons à titre d'application de ces abaques quelques résultats de mesures.

1.2. Rappel théorique. - 1.2.1. Principe de la méthode de mesure: La méthode dite de la ligne " court-circuitée " consiste essentiellement à faire réfléchir, sur un échantillon placé contre un courtcircuit, une onde électromagnétique en propagation guidée. Des caractéristiques du train d'ondes sta- tionnaires obtenues à l'avant de l'échantillon (TOST et position d'un minimum $d_{\mathrm{m}}$ ) on déduit les parties réelle et imaginaire de la constante diélectrique $\varepsilon=\varepsilon^{\prime}-j \varepsilon^{\prime \prime}$ de la substance étudiée.

1.2.2. Schéma du dispositif de mesure:

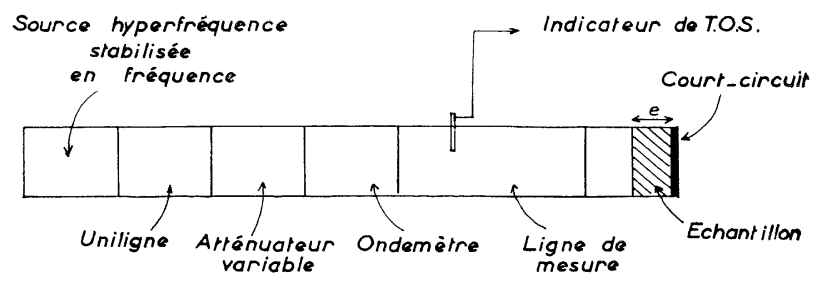

FIg. 1.--Schéma d'un banc de mesures hyperfréquences.

1.2.3. Les calculs : Les équations de propagation d'une onde électromagnétique guidée pour:le mode $\mathrm{TE}_{01}$ permettent par des reports d'impédances de lier implicitement $\varepsilon$ au TOST, $\theta$ et à $d_{\mathrm{m}}{ }^{(1)}$ pour une épaisseur d'échantillon $(e)$ et une fréquence $\left(N_{0}\right)$ donnés, par la relation (A) :

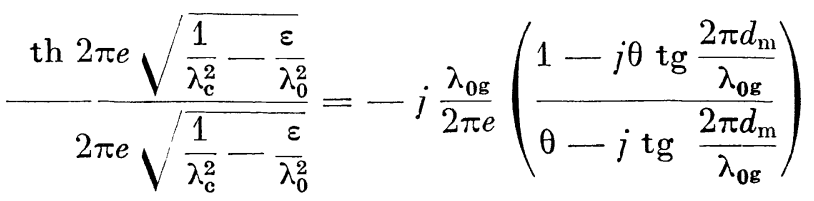

(1) Définition du $d_{\mathrm{m}}$ adopté sur nos abaques:

" $d_{\mathrm{m}}$ est le déplacement d'un minimum du train d'ondes stationnaires vers la charge, par rapport à la position qu'occuperait ce minimum si le court-circuit était sur la face d'entrée de l'échantillon." 
dans laquelle :

$\lambda_{\mathrm{c}}=$ longueur d'onde de coupure pour le guide utilisé ;

$\lambda_{\mathrm{o}}=$ longueur d'onde dans l'espace libre ;

$\lambda_{\mathrm{og}}=$ longueur d'onde guidée.

Nous ne donnons pas la démonstration de cette relation mais nous renvoyons aux différents auteurs [2], [4].

Le mode de résolution habituel de cette équation complexe est le suivant :

A l'aide du diagramme de Smith, les données expérimentales $\theta$ et $d_{\mathrm{m}}$ permettaient de calculer les parties réelle et imaginaire du second membre. Puis les abaques de Roberts, ou de Le Bot et Le Montagnier ou de Lebrun permettaient de résoudre l'équation de la forme :

$$
\text { th } A e^{\mathrm{j} \varphi} / A e^{\mathrm{j} \varphi}=B e^{\mathrm{j} \chi}
$$

et à l'aide de formules appropriées, on pouvait remonter aux valeurs de $\varepsilon^{\prime}$ et $\varepsilon^{\prime \prime}$. Mais la multiplicité de la fonction initiale nécessitait plusieurs mesures afin de lever l'indétermination.

Ce procédé de calcul était long et fastidieux et ne permettait en aucune façon d'apprécier l'incertitude de mesure sur $\varepsilon^{\prime}$ et $\varepsilon^{\prime \prime}$.

1.3. Abaques de RÉSOlution : $\left(\theta, d_{\mathrm{m}}\right) \Rightarrow\left(\varepsilon^{\prime}, \varepsilon^{\prime \prime}\right)$ pour une fréquence et une épaisseur d'échantillon données.

Nous avons programmé la relation (A) dans le langage Fortran et à l'aide d'un ordinateur I. B. M. 1620 nous avons fait les calculs nécessaires au tracé des abaques. Pour cela nous entrions $\varepsilon^{\prime}$ et $\varepsilon^{\prime \prime}$ dans l'ordinateur qui nous donnait les valeurs de $\theta$ et $d_{\mathrm{m}}$, le calcul ne pouvant se faire rapidement que dans ce sens.

Dans le but de couvrir une gamme importante de constantes diélectriques, nous avons réalisé le tracé d'une série de quatre abaques se complétant l'un l'autre par recoupement des zones de mesures. Chaque abaque, comme on va le voir, est caractérisé par son épaisseur d'échantillon et ses performances en $\varepsilon^{\prime}$ et $\varepsilon^{\prime \prime}$ consignées dans le tableau suivant :

$$
\begin{aligned}
& \text { Épaisseur } \\
& \text { EN mim VALEURS DE }: \varepsilon^{\prime} \text { VAlEuRs DE }: \varepsilon^{\prime \prime} \\
& \text { (9 } 455 \mathrm{MHz} \text { ) } \\
& \begin{array}{lrl}
e=5,50 & 1<\overline{\varepsilon^{\prime}}<6 & 0,01<\overline{\varepsilon^{\prime \prime}}<1,30 \\
e=3,00 & 5<\varepsilon^{\prime}<11 & 0,01<\varepsilon^{\prime \prime}<8 \\
e=2,00 & 10<\varepsilon^{\prime}<25 & 0,01<\varepsilon^{\prime \prime}<15 \\
e=1,00 & 20<\varepsilon^{\prime}<120 & 0,01<\varepsilon^{\prime \prime}<30
\end{array}
\end{aligned}
$$

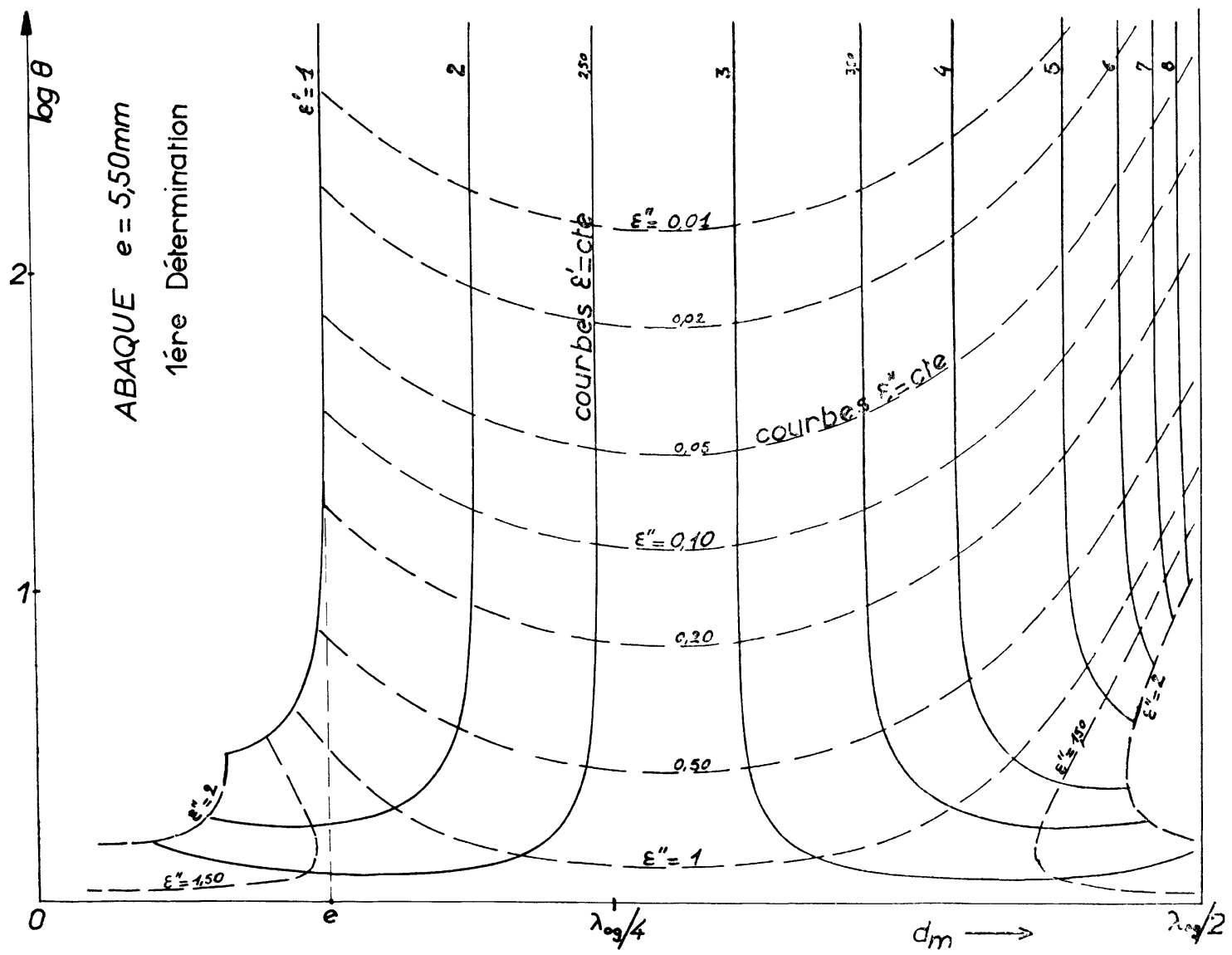

Fig. 2. - Schéma général d'un abaque. 
Présentation d'un abaque : Nous avons tracé les courbes $\varepsilon^{\prime}=$ Cte, $\varepsilon^{\prime \prime}=$ Cte dans un système cartésien de coordonnées semi-logarithmiques en prenant comme échelle :

- en ordonnée : trois modules logarithmiques de $250 \mathrm{~mm}$ chacun pour $\theta$ variant de 1 à 600 ;

- en abscisse : une échelle linéaire pour $d_{\mathrm{m}}$ variant de 0 à $\lambda_{\mathrm{og} / 2}$ représentant la première détermination de la fonction (A) $\left(^{2}\right)$.

Nous donnons ( $f i g .2$ ) le schéma simplifié de l'abaque correspondant à $e=5,50$. L'ensemble des abaques énumérés plus haut a été soigneusement tracé en détail au laboratoire pour pouvoir être utilisé à des mesures de précision.

1.4. Utilisation Des abaques. - 1.4.1. Recherche systématique d'une constante: Exemple à $9455 \mathrm{MHz}$.

Une mesure préalable sur un échantillon de $e=1 \mathrm{~mm}$ d'épaisseur permet d'avancer une valeur de $\varepsilon^{\prime}$ comprise entre $\varepsilon^{\prime}=1$ et $\varepsilon^{\prime}=120$. Si ceite valeur se trouve être inférieure à $\varepsilon^{\prime} \simeq 20$, une seconde mesure sur un échantillon de $e=2 \mathrm{~mm}$ s'impose pour préciser la valeur de $\varepsilon^{\prime}$ comprise entre $\varepsilon^{\prime}=1$ et $\varepsilon^{\prime}=20$. Si cette valeur se trouve inférieure à $\varepsilon^{\prime} \simeq 10$ une troisième mesure sur un échantillon de $e=3 \mathrm{~mm}$ permet de préciser la valeur entre $\varepsilon^{\prime}=5$ et $\varepsilon^{\prime}=11$, etc...

Mais en pratique, lorsque l'on connaît l'ordre de grandeur de $\varepsilon^{\prime}$, l'utilisation d'un seul abaque est suffisante et la taille d'une seule lame est requise.

1.4.2. Évaluation de l'incertitude: Elle est liée essentiellement aux incertitudes de mesure de $\theta$ et de $d_{\mathrm{m}}$ lorsque l'on sait s'affranchir de la taille de l'échantillon et de son épaisseur en particulier $(\Delta e \simeq 1 / 100 \mathrm{~mm})$ et de la variation de la fréquence $\left(\Delta N_{0} \simeq 100 \mathrm{kHz}\right.$ pour $\left.9455 \mathrm{MHz}\right)$.

Incertitude sur $d_{\mathrm{m}}$ : Elle peut varier selon le maté- riel (qualité de la ligne de mesure) et l'habileté de l'opérateur :

$$
0,02 \leqslant \Delta d_{\mathrm{m}} \leqslant 0,10 \mathrm{~mm}
$$

ce qui justifie le choix de l'échelle en $d_{\mathrm{m}}$ sur les abaques.

Incertitude $\operatorname{sur} \theta:$ Le problème de la mesure des TOST élevés a été envisagé afin d'atteindre avec les abaques des $\varepsilon^{\prime \prime}$ faibles; il est très complexe et nous renvoyons les lecteurs à la littérature de base [1], [8], [9].

Il nous paraît intéressant de rappeler dans cet article une correction indispensable de TOST simple, permettant d'apprécier des TOST supérieurs à 100 avec du matériel classique.

Nous proposons la formule suivante qui donne satisfaction tout en restant en accord avec celles proposées par les auteurs :

$$
\frac{1}{\theta_{\mathrm{r}}}=\frac{1}{\theta_{\mathrm{m}}}-\frac{1}{\theta_{\mathrm{cc}}}
$$

$\theta_{\mathrm{r}}=$ TOST réel

$\theta_{\mathrm{m}}=$ TOST mesuré avec l'échantillon

pour la même position

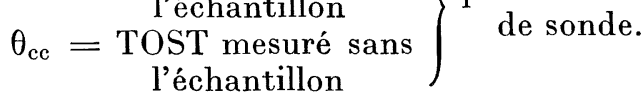

La formule n'est applicable que si $\theta_{\mathrm{m}}$ et $\theta_{\mathrm{cc}}$ sont mesurés au même niveau de la ligne de mesure. Cela implique donc une interpolation pour $\theta_{\mathrm{cc}}$ sur un diagramme préalablement tracé et dépendant de la ligne de mesure et de la cellule de mesure associée.

Enfin, cette formule suppose des mesures de $\theta_{\mathrm{m}}$ et $\theta_{\text {cc }}$ correctes, c'est-à-dire effectuées avec un cristal détecteur fonctionnant dans une zone quadratique, que ce soit au moyen d'un galvanomètre sensible ou d'un indicateur de TOST. Dans le cas où ces mesures de TOST sont effectuées à l'aide d'un atténuateur variable calibré, celui-ci devra

$$
\begin{aligned}
& \text { Ébonite } \\
& \text { - } \\
& e=5,50 \mathrm{~mm} \\
& d_{\mathrm{m}}=-9,38 \mathrm{~mm} \\
& \begin{array}{l}
\theta_{\mathrm{m}}=22 \\
\theta_{\mathrm{cc}}=40
\end{array} \\
& d_{\mathrm{m}}=+3,80 \mathrm{~mm} \\
& e=3,00 \mathrm{~mm} \\
& \theta_{\mathrm{m}}=33 \\
& \theta_{\mathrm{cc}}=36
\end{aligned}
$$

l'être parfaitement et sera du type à lame tournante par exemple.

(2) Le choix de la première détermination s'imposait pour plusieurs raisons dont les principales sont les suivantes :

10 Aux fortes constantes diélectriques on dispose en général de peu de matière (homogène et isotrope) donc d'une épaisseur faible d'échantillon.

$2^{\circ}$ Le développement excessif de la gamme des $\varepsilon^{\prime}$ à partir de la seconde détermination, nécessite un trop grand nombre d'ahaques.

\section{Avec connections}

$\varepsilon^{\prime}=2,85$

$\varepsilon^{\prime \prime}=0,060$

$\theta_{\mathbf{r}}=49$

$\varepsilon^{\prime}=2,85$

$\varepsilon^{\prime}=2,85$

$\varepsilon^{\prime \prime}=0,37$

$\theta_{\mathbf{r}}=\stackrel{n}{=} 400$

$=2,85$

$\varepsilon^{\prime \prime}=0,030$

Cette correction faite, on peut apprécier des TOST élevés donc des valeurs de $\varepsilon^{\prime \prime}$ faibles. On montre que l'incertitude relative sur $\varepsilon^{\prime \prime}$ est la même que celle $\operatorname{sur} \theta$ :

$$
\Delta \varepsilon^{\prime \prime} / \varepsilon^{\prime \prime} \simeq \Delta \theta / \theta .
$$

1.5. Exemple d'application. - Nous donnons un exemple de résultats expérimentaux montrant la nécessité de l'application de cette formule de correction de TOST pour la mesure de $\varepsilon^{\prime \prime}$ pour la fréquence $9455 \mathrm{MHz}$. 
Ces abaques étant particulièrement bien adaptés à des études de variation de permittivité complexe de mélanges, nous donnons (fig. 3 ) les résultats d'une étude d'un mélange de céramique à base de $\mathrm{TiO}_{3} \mathrm{Ba}$ en poudre et de colle araldite durci à $80^{\circ} \mathrm{C}$, les mesures étant faites à la température de $20^{\circ} \mathrm{C}$ sur des plaquettes de 1,2 , et $3 \mathrm{~mm}$ à différents pourcentages.

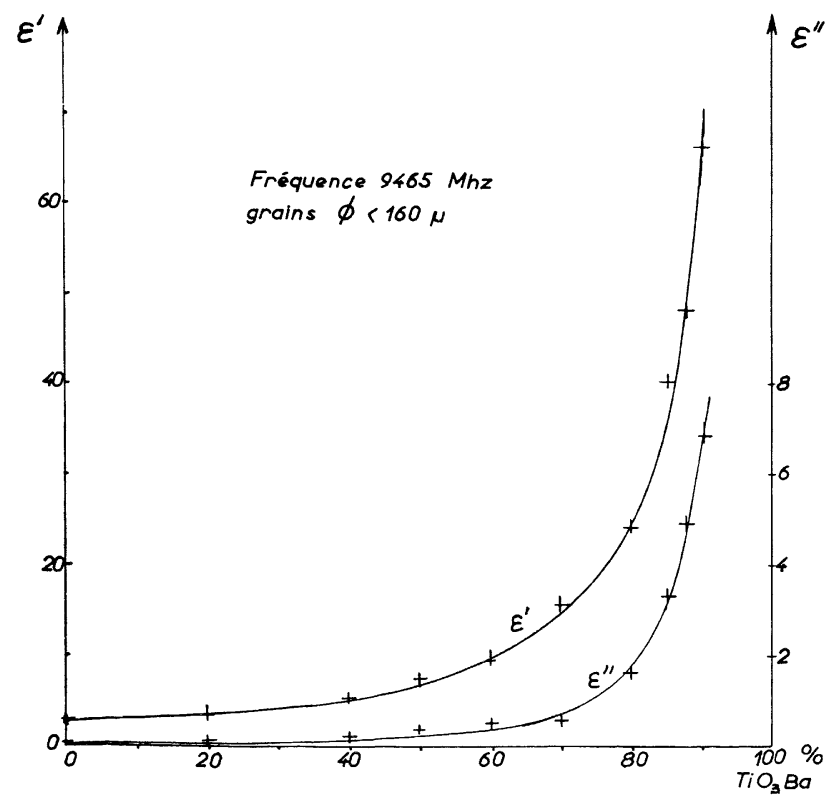

Fig. 3. -.. Étude de la permittivité complexe du mélange : Céramique $\mathrm{Ti}_{3} \mathrm{O}_{\mathrm{a}}$ en poudre-araldite.

2. Séparation et affinement des mesures de $\epsilon^{\prime}$ et $\epsilon^{\prime \prime}$. - 2.1. Introduction. - Les abaques que nous venons de présenter correspondent à la première détermination de la fonction (A) et sont destinés à un usage rapide courant ne requérant qu'une précision moyenne $(5 \%)$. Cependant, il peut être intéressant dans certains cas de déterminer avec plus de précision $\varepsilon^{\prime}$ et surtout de pouvoir évaluer de faibles $\varepsilon^{\prime \prime}$. Ceci est possible en prenant des épaisseurs d'échantillon plus importantes. On pourrait donc concevoir et réaliser des séries d'abaques pour différentes valeurs d'épaisseurs et à différentes déterminations. Le déploiement considérable de surface de papier que cela exigerait ne peut être envisagé d'une manière pratique. C'est la raison pour laquelle nous avons été amenés à réaliser un subterfuge en traçant plusieurs diagrammes. Mais néanmoins, la quantité de matière disponible (homogène et isotrope) impose une limite à l'augmentation de l'épaisseur.

2.2. Principe de la séparation des mesures de $\varepsilon^{\prime}$ et $\varepsilon^{\prime \prime}$ : L'étude de l'équation (A) et l'observation du schéma général d'un abaque ( fig. 2) à une détermination quelconque, nous a permis d'apporter les conclusions suivantes relatives à la zone centrale :
2.2.1. Les courbes $\varepsilon^{\prime}=$ Cte sont parallèles entre elles et à l'axe des TOST pour une largeur en $d_{m}$ importante et pour des valeurs de TOST allant de l'infini à une valeur faible (2 ou 3 ) suivant l'écart en $d_{\mathrm{m}}$.

2.2.2. Les courbes $\varepsilon^{\prime \prime}=$ Cte sont sensiblement normales aux précédentes pour le même écart en $d_{\mathrm{m}}$ (rigoureusement pour $\varepsilon^{\prime}=\varepsilon_{\mathrm{c}}^{\prime}$ ) (fig. 4). En choisissant une échelle logarithmique pour les TOST, ces courbes se répartissent elles aussi suivant une échelle logarithmique de même module mais d'ordre croissant inversé. Le long de la courbe $\varepsilon_{c}^{\prime}=$ Cte on peut écrire la relation (B) :

$$
\varepsilon^{n}=\varepsilon_{\mathrm{c}}^{\prime \prime} / \theta \text {. }
$$

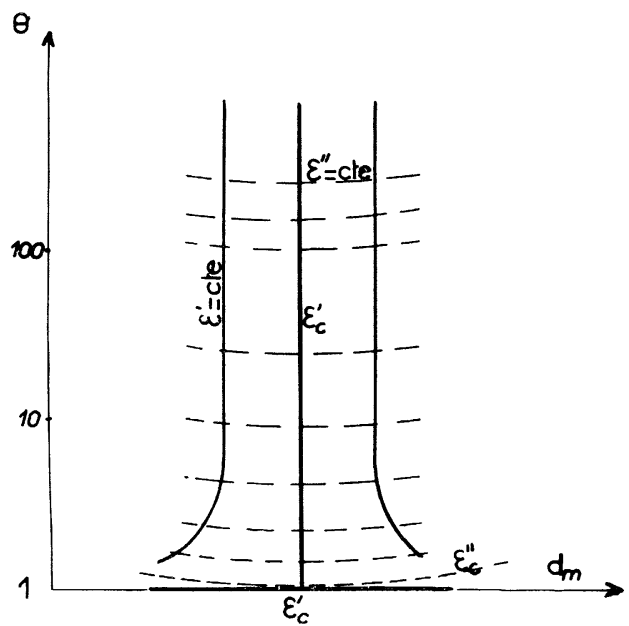

Fig. 4. - Schéma de la zone centrale d'un abaque indiquant la valeur caractéristique $\varepsilon_{\mathrm{c}}=\varepsilon_{\mathrm{c}}^{\prime}-\mathrm{J} \varepsilon_{\mathrm{c}}^{\prime \prime}$.

Ces observations permettent de séparer les mesures de $\varepsilon^{\prime}$ et $\varepsilon^{\prime \prime}$.

2.3. Diagrammes de résolution pour $\varepsilon^{\prime}$. 2.3.1. Description : Ils ont été tracés à la première et à la troisième détermination de la fonction (A), pour $\varepsilon^{\prime \prime}=0$ (donc $\theta=\infty$ ) dans un système de coordonnées rectangulaires $\left(e, d_{\mathrm{m}}\right)$, et se traduisent par des courbes $\varepsilon^{\prime}=$ Cte, pour une variation de $d_{m}$ faible autour de la zone centrale ( $f$ ig. 5 ).

On a tracé sur ce diagramme une ligne centrale coupant les courbes $\varepsilon^{\prime}=$ Cte et matérialisant la valeur de $\varepsilon_{\mathrm{c}}^{\prime}$ pour chaque épaisseur.

2.3.2. Utilisation: Connaissant l'ordre de grandeur d'une constante diélectrique $\varepsilon_{1}^{\prime}$, l'intersection de la ligne centrale et de la courbe $\varepsilon_{1}^{\prime}=$ Cte définit l'épaisseur optimum d'échantillon (fig. 5). Si le point expérimental tombe dans le diagramme, la mesure est suffisante, sinon une extrapolation est possible et on peut ramener la valeur $\varepsilon_{1}^{\prime}$ au centre $d u$ diagramme en modifiant l'épaisseur.

Théoriquement, la précision des mesures augmente en passant à des déterminations d'ordre supé- 


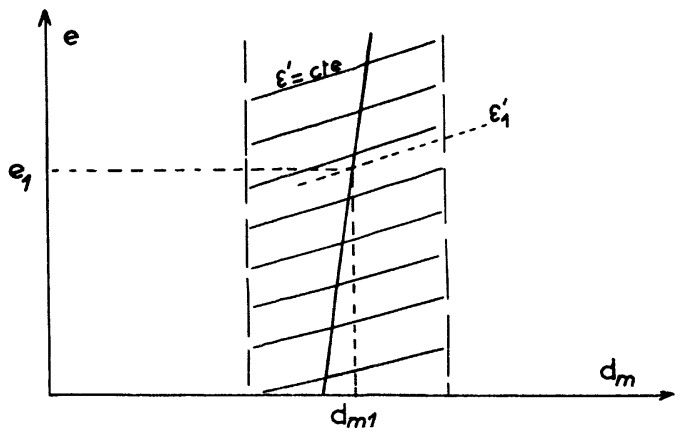

Frg. 5. - Schéma et utilisation du diagramme $e=f\left(d_{\mathrm{m}}\right)$

rieur. La limite est imposée à la fois par la quantité de matière disponible (homogène et isotrope) et par la valeur de l'absorption $\left(\varepsilon^{\prime \prime}\right)$.

2.4. Diagrammes de Résolution pour $\varepsilon^{\prime \prime}$. 2.4.1. Description: Ils ont été tracés pour les dix premières déterminations de la fonction (A) pour $\theta=1$ dans un système de coordonnées rectangulaires. Ce sont les courhes $e=f\left(\varepsilon_{\mathrm{c}}^{\prime}\right)$ et $\varepsilon_{\mathrm{c}}^{\prime \prime}=f\left(\varepsilon_{\mathrm{c}}^{\prime}\right)$ (fig. 6). $\varepsilon_{\mathrm{c}}=\varepsilon_{\mathrm{c}}^{\prime}-j \varepsilon_{\mathrm{c}}^{\prime \prime}$ est la valeur de la constante diélectrique au centre d'un abaque (fig. 4).

2.4.2. Utilisation : Connaissant $\varepsilon=\varepsilon_{1}^{\prime}-j \varepsilon_{1}^{\prime \prime}$ une constante diélectrique mesurée à l'aide des abaques à la première détermination, et pour laquelle $\varepsilon_{1}^{\prime \prime}$ est entaché d'une incertitude trop grande, du fait de sa faible valeur, donc de sa mesure avec un TOST élevé imprécis, le but de ces diagrammes est d'améliorer cette mesure de $\varepsilon_{1}^{\prime \prime}$.

Traçons dans le diagramme donné ( $f g .6$ ) la droite $\varepsilon_{1}^{\prime}=$ Cte : c'est une droite parallèle à l'axe des ordonnées. Son intersection avec les deux réseaux de courbes nous donne pour chaque détermination respectivement les épaisseurs d'échantillon utilisables et l'origine de l'échelle logarithmique des TOST par rapport à l'échelle logarithmique des $\varepsilon^{\prime \prime}$.

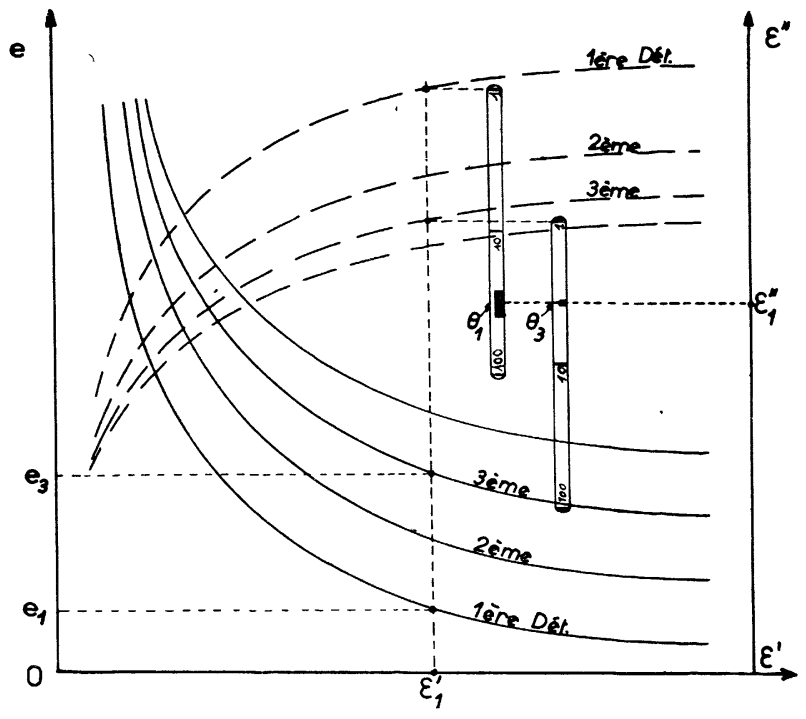

FIG. 6. - Schéma et utilisation des diagrammes $e=t\left(\varepsilon^{\prime}\right), \quad \varepsilon^{\prime \prime}=f\left(\varepsilon^{\prime}\right)$.

En pratique on utilise une réglette logarithmique de même module que celui de l'échelle des $\varepsilon^{\prime \prime}$ pour le report des TOST.

On a fait figurer aux niveaux des TOST, $\theta_{1}$ et $\theta_{3}$ les plages d'incertitude, ce qui permet d'orienter le choix des épaisseurs (fig. 6).

3. Extension de l'emploi des abaques à d'autres fréquences. - Nous avons exposé une étude complète pour la fréquence de $9455 \mathrm{MHz}$. Mais ces abaques et diagrammes restent valables pour d'autres fréquences.

Nous donnons les correspondances épaisseurfréquence pour des équipements de mesures hyperfréquences utilisant du matériel standard guide [11] dans le tableau suivant :

\begin{tabular}{|c|c|c|c|c|c|c|}
\hline $\begin{array}{c}\text { BANDE } \\
\text { DE FRÉQUENCE }\end{array}$ & $\begin{array}{l}\lambda_{c} \\
\mathrm{~mm}\end{array}$ & $k=\frac{\lambda_{c X}}{\lambda_{c}}$ & $\begin{array}{c}N=\frac{N_{X}}{k} \\
\mathrm{MHz}\end{array}$ & $\begin{array}{c}e=k e_{X} \\
\mathrm{~mm}\end{array}$ & $d_{\mathrm{mm}}=\frac{d_{\mathrm{m} X}}{k}$ & $\theta=\theta_{X}$ \\
\hline $\bar{X}$ & $\overline{45,72}$ & $\overline{1}$ & $\overline{9455}$ & $\overline{5,50}$ & $\overline{d_{\mathrm{m} X}}$ & $\overline{\theta_{X}}$ \\
\hline$S$ & 144,28 & 3,156 & 3000 & 16,49 & $\frac{d_{\mathrm{m} x}}{3,156}$ & $\theta_{X}$ \\
\hline$Q$ & 14,22 & 0,311 & 30432 & 1,71 & $\frac{d_{\mathrm{m} X}}{0,311}$ & $\theta_{X}$ \\
\hline
\end{tabular}

4. Conclusion. - Nous avons présenté dans la première partie de cet article un système d'abaques permettant d'obtenir par simple lecture la constante diélectrique complexe à partir des données expérimentales et d'évaluer en même temps, dans chaque cas, une incertitude représentant une limite supérieure de l'erreur de mesure.
Dans la seconde partie, la méthode d'affinement et de séparation des mesures a été mise au point dans le but de pouvoir mesurer de très faibles valeurs absolues d'absorptions $\left(\varepsilon^{\prime \prime}\right)$.

Manuscrit reçu le 9 juillet 1916 . 


\section{BIBLIOGRAPHIE}

[1] Montgomery (G.), Technique des mesures en micro-ondes. Tome II. Éd. Langue Française, Chiron, 1953, pp. 584-700.

[2] Von Hippel (A. R.), Les diélectriques et leurs applications, Dunod, 1961, pp. 45-121.

[3] Rapaport (H.), Handbook of microwave measurements. Vol. 1, Sect. X. Pol. Press of Brooklyn, New York, 1958.

[4] Raoult (G.), Les ondes centimétriques, Masson, 1958, pp. 193-216.

[5] Roberts (S.) et Von Hippel (A. R.), Appl. Physics, 1946, 17, 610 .
[6] Le Bot (J.), Thèse, Paris, 1953.

Le Montagner (S.), Thèse, Paris, 1954.

[7] Le brun (A.), Thèse, Lille, 1953.

[8] Grnzton (E. L.), Microwave measurements, MacGraw-Hill Book Company Inc., New York, 1957, pp. 235-312.

[9] Raoult (G.), Les ondes centimétriques, Masson, 1958, pp. $90-104$.

[10] Demau (C.) et Derbos (G.), C. R. Acad. Sc., 1965, 261, 5397-5399.

[11] Harvey (A. F.), Microwave engineering, Acad. Press, London and New York, 1963, p. 50. 